\title{
Matched Comparison of Fusion Rates between Hydroxyapatite Demineralized Bone Matrix and Autograft in Lumbar Interbody Fusion
}

\author{
Dae Hwan Kim, M.D., Nam Lee, M.D., Ph.D., Dong Ah Shin, M.D., Ph.D., Seong Yi, M.D., Ph.D., Keung Nyun Kim, M.D., Ph.D., \\ Yoon Ha, M.D., Ph.D. \\ Department of Neurosurgery, Spine and Spinal Cord Institute, Yonsei University College of Medicine, Seoul, Korea
}

Objective : To compare the fusion rate of a hydroxyapatite demineralized bone matrix (DBM) with post-laminectomy acquired autograft in lumbar interbody fusion surgery and to evaluate the correlation between fusion rate and clinical outcome.

Methods : From January 2013 to April 2014, 98 patients underwent lumbar interbody fusion surgery with hydroxyapatite DBM (HA-DBM group) in our institute. Of those patients, 65 received complete CT scans for 12 months postoperatively in order to evaluate fusion status. For comparison with autograft, we selected another 65 patients who underwent lumbar interbody fusion surgery with post-laminectomy acquired autograft (Autograft group) during the same period. Both fusion material groups were matched in terms of age, sex, body mass index (BMI), and bone mineral density (BMD). To evaluate the clinical outcomes, we analyzed the results of visual analogue scale (VAS), Oswestry Disability Index (ODI), and Short Form Health Survey (SF-36).

Results : We reviewed the CT scans of 149 fusion levels in 130 patients (HA-DBM group, 75 levels/65 patients; Autograft group, 74 levels/65 patients). Age, sex, BMl, and BMD were not significantly different between the groups ( $p=0.528, p=0.848, p=0.527$, and $p=0.610$, respectively). The HA-DBM group showed 39 of 75 fused levels (52\%), and the Autograft group showed 46 of 74 fused levels (62.2\%). This difference was not statistically significant $(p=0.21)$. In the HA-DBM group, older age and low BMD were significantly associated with non-fusion (61.24 vs. $66.68, p=0.027$; -1.63 vs. $-2.29, p=0.015$, respectively). VAS and ODI showed significant improvement after surgery when fusion was successfully achieved in both groups ( $p=0.004, p=0.002$, HA-DBM group; $p=0.012, p=0.03$, Autograft group).

Conclusion : The fusion rates of the hydroxyapatite DBM and Autograft groups were not significantly different. In addition, clinical outcomes were similar between the groups. However, older age and low BMD are risk factors that might induce non-union after surgery with hydroxyapatite DBM.

Key Words : Hydroxyapatite · Autograft · Fusion · CT scan.

\section{INTRODUCTION}

Spinal fusion is the most frequently performed surgery to treat spinal disorders. The ultimate aim of spinal fusion surgery is to achieve complete fusion at the index level. Non-union is a critical complication of this surgery, with a prevalence of approximately $10 \%$ to $40 \%{ }^{21)}$.

Equipment such as pedicle screws, rods, wires, and cages have been used to achieve fusion of unstable spinal segments. However, previous studies have reported that $10 \%$ to $15 \%$ of all such cases showed pseudoarthrosis ${ }^{21)}$. Bone grafting is another strategy to augment spinal fusion. Autologous iliac bone graft is the most commonly used bone graft and is regarded as the gold standard in bone grafting ${ }^{21)}$. This method is preferred due to successful fusion rate and low risk of disease transmission ${ }^{11)}$. However, this procedure is sometimes associated with unexpected morbidities such as fractures, hernia, ureteral injury, pelvic instability, infection, and chronic donor site pain ${ }^{21)}$. In addition, there are several limitations aside from its morbidity including separate incision for harvesting, increased surgical time, and limited or insufficient amount of graft.

Demineralized bone matrix (DBM), which is produced from human allograft tissue, is an alternative to an autograft. DBM consists of bone morphogenic proteins (BMP), collagen, and other synergistic proteins such as transforming growth factor and insulin-like growth factor ${ }^{21)}$. Therefore, DBM has osteoin-

- Received : November 9, 2015 • Revised : January 31, 2016 •Accepted : February 26, 2016

- Address for reprints : Yoon Ha, M.D., Ph.D.

Department of Neurosurgery, Yonsei University College of Medicine, 50 Yonsei-ro, Seodaemun-gu, Seoul 03722, Korea

Tel : +82-2-2228-2150, Fax : +82-2-393-9979, E-mail : hayoon@yuhs.ac

- This is an Open Access article distributed under the terms of the Creative Commons Attribution Non-Commercial License (http://creativecommons.org/licenses/by-nc/3.0) which permits unrestricted non-commercial use, distribution, and reproduction in any medium, provided the original work is properly cited. 
ductive and osteoconductive characteristics that allow it to be used as a graft extender or substitute ${ }^{21)}$. Hydroxyapatite is also frequently used as a graft extender or substitute because it has osteoinductive characteristics and augmentation of the osteoconductive features of an autograft ${ }^{25}$. According to prior investigations, hydroxyapatite shows nearly equivalent fusion results to autografts ${ }^{1,23,25)}$.

Many trials have been conducted on the surgical outcomes of spinal fusion surgery using allografts. Reportedly, in many trials, the fusion rates of DBM were similar to those of autografts. Cammisa et al. ${ }^{8)}$ reported a 52\% fusion rate in Grafton DBM in lumbar spinal surgery, which is comparable to $54 \%$ when using autografts. An et al. ${ }^{2}$ reported a 54\% fusion rate in anterior cervical fusion surgery using Grafton DBM and 74\% when using autografts. However, many of these studies evaluated the fusion status using plain radiographs.

Hydroxyapatite DBM is a newly designed material, the fusion rate of which has not yet been reported. In this study, we investigated and compared the fusion rates of hydroxyapatite DBM and autografts using CT scans at 12 months after operation. In addition, we measured clinical scores to evaluate the impact of fusion on clinical outcomes.

\section{MATERIALS AND METHODS}

\section{Patient population}

From January 2013 to April 2014, 98 patients diagnosed with lumbar stenosis underwent lumbar interbody fusion surgery using hydroxyapatite DBM (Bonfuse ${ }^{\mathrm{TM}}$, CG Bio, Seoul, Korea) in Severance Hospital. This study was approved by the institutional review boards at Severance Hospital. We excluded patients with a history of infection, trauma, spinal tumor, spondylolytic spondylolisthesis, or previous spinal surgery. Patients using steroid medications or who had metabolic bone disease such as Paget's disease or renal osteodystrophy were also excluded. Ultimately, 65 patients (75 interbody levels) were eligible for this study and underwent postoperative CT scans for 12 months (the HA-DBM group). Another 65 patients (74 interbody levels) who underwent lumbar interbody fusion surgery using autograft during the same period were enrolled for comparison (the Autograft group). The subjects in the Autograft group were of similar age, sex, bone mineral density (BMD), and body mass index (BMI) and also underwent postoperative CT scans for 12 months. Therefore, a total of 130 patients (149 interbody levels) were evaluated using CT scans.

\section{Operative technique}

One fusion technique [posterior lumbar interbody fusion, (PLIF) anterior lumbar interbody fusion (ALIF) or transforaminal lumbar interbody fusion (TLIF)] was performed in each patient. We performed pedicle screw fixation on each PLIF and TLIF case. All of TLIF cases were open TLIF and minimal invasive TLIF was not conducted. The DBM volume was deter- mined by the size of the cage. Each surgical technique requires an appropriate cage in terms of height, length, angle, and shape. Therefore, we used the optimal volume of DBM allograft in each case. When using autologous bone graft, the extracted lamina and spinous process were cut into small pieces and used to fill the cage.

\section{Assessment of fusion state}

Fusion grade was assessed using coronal or sagittal reconstruction images of lumbar CT scans. All CT scans were performed at one year after surgery. The fusion grade was classified from Grade I to IV ${ }^{24)}$. We classified Grade I (cortical union of the graft material and central trabecular continuity) and Grade II (cortical union of the structural graft material with partial trabecular incorporation) as "Fused" and Grade III (superior or inferior cortical non-union of the central graft material with partial trabecular discontinuity centrally) and Grade IV (both superior and inferior cortical non-union with a complete lack of central trabecular continuity) as "Not-fused" (Fig. 1).

We also measured clinical outcomes including visual analogue scale (VAS) score, Oswestry Disability Index (ODI), and Short Form Health Survey (SF-36) score in order to compare the surgical outcomes between the two groups.

\section{Measurement validation}

To assess the reliability of the measurements, two examiners measured the fusion status twice at a one-week interval for each of the 130 cases. In cases of discrepancies, the fusion grade was determined through debate in conferences. Inter-examiner and intra-examiner ICC and 95\% confidence interval (95\% CI) were assessed. Inter-examiner reliability was assessed using the intra-class correlation coefficient (ICC) of data obtained for the measurement of fusion status between both examiners. Intraexaminer reliability was assessed between the first and second measurements for each individual examiner.

\section{Statistical analysis}

The measured variables were analyzed using independent $\mathrm{t}$ test analysis and chi-square analysis. We applied the paired t-

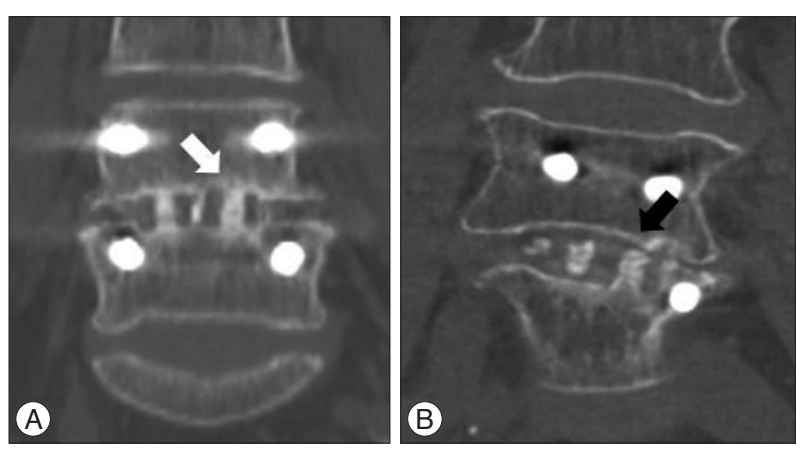

Fig. 1. Fusion assessment using computed tomography (CT) scan. A : Fused, cortical continuity was observed without a visual gap on CT scan, white arrow. B : Not fused, cortical discontinuity was observed with a visual gap, black arrow. 
test to compare the clinical outcomes in each group. Analysis was conducted using the PASW statistics 18 software program (PASW, IBM, USA). A $p<0.05$ was considered statistically significant.

\section{RESULTS}

Mean age was not significantly different between the groups

Table 1. Group demographics

\begin{tabular}{lccc}
\hline & $\begin{array}{c}\text { HA-DBM group, } \\
\text { mean } \pm \text { SD }\end{array}$ & $\begin{array}{c}\text { Autograft group, } \\
\text { mean } \pm \text { SD }\end{array}$ & $p$-value \\
\hline Age (years) & $63.66 \pm 10.19$ & $62.54 \pm 10.04$ & 0.528 \\
Sex (M : F) & $16: 46$ & $20: 45$ & $0.848^{*}$ \\
BMD (T-score) & $-1.91 \pm 1.06$ & $-2.05 \pm 1.27$ & 0.527 \\
BMI (kg/m $\left.{ }^{2}\right)$ & $27.39 \pm 3.03$ & $25.55 \pm 3.75$ & 0.610 \\
Procedure & & & \\
$\quad$ PLIF & 36 & 47 & \\
ALIF & 3 & 0 & \\
TLIF & 36 & 27 &
\end{tabular}

Independent t-test was performed. ${ }^{*}$ Chi-square tests were performed. HA-DBM : hydroxyapatite-demineralized bone matrix allograft, BMD : bone mineral density, BMI : body mass index, PLIF : posterior lumbar interbody fusion, ALIF : anterior lumbar interbody fusion, TLIF : transforaminal lumbar interbody fusion, SD : standard deviation

Table 2. Comparison of fusion rates at postoperative 12 months

\begin{tabular}{lccc}
\hline & HA-DBM group & Autograft group & $p$-value \\
\hline Fused levels & 39 & 46 & \\
Not fused levels & 36 & 28 & \\
Fusion rate (\%) & 52 & 62.2 & $0.210^{*}$ \\
\hline
\end{tabular}

${ }^{*}$ Chi-square tests were performed to analyze the fusion rate. Fusion rate=fused levels/(fused+not fused levels) $\times 100$. HA-DBM : hydroxyapatite demineralized bone matrix
(HA-DBM group 63.66 \pm 10.19 vs. Autograft group 62.54 \pm 10.04 , $p=0.528$ ). In addition, sex ratio (HA-DBM group $16: 46 \mathrm{vs.} \mathrm{Au-}$ tograft group $20: 45, p=0.848$ ), BMD (HA-DBM group -1.91 \pm 1.06 vs. Autograft group -2.05 $\pm 1.27, p=0.527$ ), and BMI (HADBM group $27.39 \pm 3.03$ vs. Autograft group 25.55 \pm 3.75 , $p=0.610$ ) were not significantly different. The HA-DBM group contained three cases of ALIF and the Autograft group none (Table 1).

In the HA-DBM group, 39 interbody levels were "Fused" and 36 interbody levels were "Not-fused," resulting in a 52\% fusion rate. In the Autograft group, 49 interbody levels were "Fused" and 28 levels were "Not-fused," resulting in a $62.2 \%$ fusion rate. Fusion rate was not statistically significantly different between the two groups $(p=0.21)$ (Table 2$)$.

Next, we compared the "Fused" subgroup with the "Notfused" subgroup in each group. In the HA-DBM group, the "Fused" subgroup showed significantly lower mean age than the "Not-fused" subgroup ("Fused" subgroup $61.24 \pm 9.45$ vs. "Not-fused" subgroup $66.86 \pm 10.42, p=0.027)$. In addition, the BMD was significantly lower in the "Not-fused" subgroup ("Fused" subgroup $-1.63 \pm 0.90$ vs. "Not-fused" subgroup $-2.29 \pm$ $1.16, p=0.015)$. Conversely, there were no significant differences between the "Fused" and the "Not-fused" subgroups in the Autograft group (Table 3).

VAS and ODI were improved significantly in the HA-DBM group regardless of fusion (preoperative 5.3, postoperative 2.9; preoperative 40.8, postoperative 48.6 in "Fused"; preoperative 6.2, postoperative 3.4; preoperative 48.6, postoperative 29.7 in "Not-fused"). In the Autograft group, VAS and ODI showed significant improvement in the "Fused" subgroup (preoperative 5.0, postoperative 2.6 and preoperative 41.2 , postoperative 28.6 , respectively) but not in the "Not-fused" subgroup ( $p=0.192)$. The

Table 3. Comparison of demographics in terms of fusion

\begin{tabular}{|c|c|c|c|c|c|c|}
\hline & \multicolumn{3}{|c|}{ HA-DBM group } & \multicolumn{3}{|c|}{ Autograft group } \\
\hline & Fused, mean \pm SD & Not fused, mean \pm SD & $p$-value & Fused, mean \pm SD & Not fused, mean $\pm S D$ & $p$-value \\
\hline Age (years) & $61.24 \pm 9.45$ & $66.86 \pm 10.42$ & 0.027 & $61.71 \pm 11.06$ & $63.7 \pm 8.46$ & 0.435 \\
\hline $\operatorname{Sex}(M: F)$ & $9: 28$ & $10: 18$ & $0.317^{\star}$ & $14: 24$ & $6: 21$ & $0.208^{\star}$ \\
\hline BMD (T-score) & $-1.63 \pm 0.90$ & $-2.29 \pm 1.16$ & 0.015 & $-2.08 \pm 1.39$ & $-2.01 \pm 1.12$ & 0.855 \\
\hline $\operatorname{BMI}\left(\mathrm{kg} / \mathrm{m}^{2}\right)$ & $23.72 \pm 3.22$ & $24.04 \pm 2.80$ & 0.675 & $25.59 \pm 3.83$ & $25.48 \pm 3.70$ & 0.908 \\
\hline
\end{tabular}

Independent t-test was performed. ${ }^{*}$ Chi-square tests were performed. HA-DBM : hydroxyapatite demineralized bone matrix, BMD : body mass index, BMI : bone mineral density

Table 4. Comparison of clinical outcomes between groups

\begin{tabular}{|c|c|c|c|c|c|c|c|c|c|c|c|c|}
\hline & & & HA-D & M group & & & & & Auto & fft group & & \\
\hline & & Fused & & & Not fused & & & Fused & & & Not fused & \\
\hline & $\begin{array}{c}\text { Preoperative, } \\
\text { mean } \pm S D\end{array}$ & $\begin{array}{c}\text { Postoperative, } \\
\text { mean } \pm \text { SD }\end{array}$ & $p$-value & $\begin{array}{c}\text { Preoperative, } \\
\text { mean } \pm S D\end{array}$ & $\begin{array}{c}\text { Postoperative, } \\
\text { mean }+ \text { SD }\end{array}$ & $p$-value & $\begin{array}{c}\text { Preoperative, } \\
\text { mean } \pm \text { SD }\end{array}$ & $\begin{array}{c}\text { Postoperative, } \\
\text { mean } \pm S D\end{array}$ & le & $\begin{array}{c}\text { Preoperative, } \\
\text { mean }+ \text { SD }\end{array}$ & $\begin{array}{c}\text { Postoperative, } \\
\text { mean } \pm S D\end{array}$ & $p$ \\
\hline TAS & $5.31 \pm 2.52$ & $2.93 \pm 2.17$ & 04 & $6.16 \pm$ & $3.45 \pm$ & 0.008 & 21 & 2.56 & 0.012 & $3.56 \pm 3.62$ & $2.21 \pm 1.59$ & 0.192 \\
\hline DI & $40.8 \pm 15.5$ & $22.3 \pm 19.3$ & 0.002 & $48.6 \pm 21.7$ & $29.7 \pm 16.3$ & & & $28.6 \pm 20.3$ & 0.030 & $36.0 \pm 14.4$ & $25.8 \pm 15.5$ & 0.149 \\
\hline CS & $55.7 \pm 11.8$ & $51.9 \pm 6.3$ & 0.455 & $51.6 \pm 2.8$ & $53.4 \pm 1.4$ & 0.626 & $53.8 \pm 6.5$ & $56.7 \pm 19.9$ & 0.528 & $54.5 \pm 6.2$ & $45.9 \pm 6.9$ & 0.885 \\
\hline ICS & $51.4 \pm 18.4$ & $54.9 \pm 14.4$ & 0.302 & $58.3 \pm 21.2$ & $60.5 \pm 17.6$ & 0.500 & $48.0 \pm 9.2$ & $57.9 \pm 16.6$ & 0.456 & $49.6 \pm 7.4$ & $58.9 \pm 13.0$ & 0.076 \\
\hline
\end{tabular}

Paired t-test was performed to analyze the clinical outcome score. VAS : visual analogue scale, ODI : Oswestry Disability Index, PCS : physical component score, MCS : mental component score 
SF-36 score, which was composed of a physical component score (PCS) and a mental component score (MCS), was not significantly different between the groups regardless of fusion (Table 4).

\section{DISCUSSION}

Spinal fusion surgery is the treatment of choice in various spinal disorders. Pseudoarthrosis has remained a challenging problem with this surgery; thus, bone grafts are used to alleviate this problem. Due to the limitations of iliac bone graft, local corticocancellous bone chip acquired from laminectomy is frequently used. The fusion results whether using local bone chip or iliac bone graft are equivalent ${ }^{15)}$. However, limited volume might results from this conventional method and can be unfavorable in some cases with a low proportion of cancellous bone in the local graft ${ }^{4}$. As an alternative, DBM products were developed to use as a graft expander or substitute. The fusion rates of previous commercial DBM products are similar to those of Autograft $^{8,13,16)}$.

The fusion achieved when using DBM and hydroxyapatite mixtures have been evaluated in several trials. According to Lee et $\mathrm{al}^{20}{ }^{20}$, the combined use of DBM and hydroxyapatite indicated osteoblastic differentiation in vitro. They showed that the mixture has osteoinductive characteristics by performing in vitro studies. In addition, many clinical trials have reported successful fusion results with hydroxyapatite $\mathrm{DBM}^{25,26)}$. Osteoinductivity and osteoconductivity were present in the hybrid compound, indicating that hydroxyapatite with DBM can be an adjunct to autografts $^{25)}$.

Our results showed a lower fusion rate in the hydroxyapatite HA-DBM group than the Autograft group, although the difference was not statistically significant. Many previous studies have reported lower fusion rates of DBM allograft than autograft, but also without statistical significance ${ }^{8,10,16}$. Although the fusion rates of DBM allograft were lower than those of autograft, the surgical outcomes of DBM allograft were not inferior to those of autograft ${ }^{8,10,16)}$, suggesting that DBM allograft is a good alternative to autograft ${ }^{21}$. The overall fusion rates in previous studies were higher than those in the present study ${ }^{8,16)}$. Fusion rates in previous articles regarding allograft material vary widely from $60 \%$ to $98 \%{ }^{7}$. We believe these differences originated from the different methods used for fusion assessment. Most previous studies assessed fusion status using plain radiographs; however, CT scans are more reliable to evaluate the exact fusion status ${ }^{9,19}$. Therefore, the fusion rate assessed using $\mathrm{CT}$ scans is more accurate and more precisely represents the fusion rate.

Unlike the Autograft group, the fusion rate in the HA-DBM group was affected by age and BMD. Okuyama et al. ${ }^{22}$ reported that pseudoarthrosis was more frequent in patients with low BMD. In addition, Inoue et al. ${ }^{14)}$ reported that bone formation is decreased in older patients due to a smaller number of stromal cells in bone marrow. In addition, in animal studies, bone healing was shown to require a longer time in older age ${ }^{5}$. Low $\mathrm{BMD}$ and older age are risk factors for non-union. However, these factors affected only the HA-DBM group in our study. This finding suggests that hydroxyapatite DBM is more susceptible to age and BMD than is autograft. Consequently, when considering spinal fusion surgery with DBM products, older age and low BMD should be taken into account as risk factors.

We measured clinical scores by evaluating VAS, ODI, and SF36 (PCS and MCS) scores. The VAS and ODI score after surgery were improved in both groups. These results are similar to other previous studies ${ }^{10,16)}$. The improvements were statistically significant except in the "Not-fused" subgroup of the Autograft group. This result might be due to the small number of enrolled patients. In addition, the changes in SF-36 score were not significant after surgery. We also believe that 12 months is a relatively short period in which to achieve complete fusion status, and bone formation and tissue healing might be ongoing at fusion sites.

This study had several limitations. First, CT scans were performed 12 months after surgery. Considering that a two-year follow-up has been proposed as the time required to definitively evaluate the solidity and stability of spine fusion ${ }^{6}, 12$ months might be too short to precisely evaluate fusion status and can lead to underestimation of fusion results. The other limitation was that we did not consider other factors that might affect fusion status. Diabetes mellitus is known to reduce the fusion rate in spinal surgery ${ }^{12}$. Diabetes delays the postoperative bone healing process stimulated by enhanced tumor necrosis factor $\alpha$ to reduce proliferation and promote necrosis of mesenchymal stem cells $^{17)}$. Additionally, local insulin concentration can affect the fusion results by modifying the level of insulin like growth factor-1 at the fusion site ${ }^{18}$. Smoking also affects bone formation. An et al. ${ }^{2)}$ and Anderson et al. ${ }^{3)}$ reported that nicotine disturbs the microcirculation of fusion beds and affects the fusion rate.

\section{CONCLUSION}

This study demonstrated that the fusion rates of a hydroxyapatite HA-DBM group and Autograft group were not significantly different. In addition, the clinical outcomes were similar between the groups. Therefore, the hydroxyapatite DBM allograft can be used as an alternative to conventional autologous bone grafts but should be carefully considered in older or lowBMD patients.

\section{- Acknowledgements}

The research was supported by a research grant (Grant No. 2013-310013) from Daewoong Pharmaceutical Co. Ltd. (Korea).

\footnotetext{
References

1. Agrillo U, Mastronardi L, Puzzilli F : Anterior cervical fusion with carbon fiber cage containing coralline hydroxyapatite : preliminary observations in 45 consecutive cases of soft-disc herniation. J Neurosurg 96 (3
} 
Suppl) : 273-276, 2002

2. An HS, Simpson JM, Glover JM, Stephany J : Comparison between allograft plus demineralized bone matrix versus autograft in anterior cervical fusion. A prospective multicenter study. Spine (Phila Pa 1976) 20 : 2211-2216, 1995

3. Andersen T, Christensen FB, Laursen M, Høy K, Hansen ES, Bünger C: Smoking as a predictor of negative outcome in lumbar spinal fusion. Spine (Phila Pa 1976) 26 : 2623-2628, 2001

4. Antoni M, Charles YP, Walter A, Schuller S, Steib JP : Fusion rates of different anterior grafts in thoracolumbar fractures. J Spinal Disord Tech 28 : E528-E533, 2015

5. Bak B, Andreassen TT : The effect of aging on fracture healing in the rat. Calcif Tissue Int $45: 292-297,1989$

6. Boden SD : Bone repair and enhancement clinical trial design. Spine applications. Clin Orthop Relat Res (355 Suppl) : S336-S346, 1998

7. Bono CM, Lee CK : Critical analysis of trends in fusion for degenerative disc disease over the past 20 years : influence of technique on fusion rate and clinical outcome. Spine (Phila Pa 1976) 29 : 455-463; discussion Z5, 2004

8. Cammisa FP Jr, Lowery G, Garfin SR, Geisler FH, Klara PM, McGuire RA, et al. : Two-year fusion rate equivalency between Grafton DBM gel and autograft in posterolateral spine fusion : a prospective controlled trial employing a side-by-side comparison in the same patient. Spine (Phila Pa 1976) 29 : 660-666, 2004

9. Carreon LY, Glassman SD, Djurasovic M : Reliability and agreement between fine-cut CT scans and plain radiography in the evaluation of posterolateral fusions. Spine J $7: 39-43,2007$

10. Dimar JR, Glassman SD, Burkus KJ, Carreon LY : Clinical outcomes and fusion success at 2 years of single-level instrumented posterolateral fusions with recombinant human bone morphogenetic protein-2/compression resistant matrix versus iliac crest bone graft. Spine (Phila Pa 1976) 31 : 2534-2539; discussion 2540, 2006

11. Finkemeier CG : Bone-grafting and bone-graft substitutes. J Bone Joint Surg Am 84-A : 454-464, 2002

12. Glassman SD, Alegre G, Carreon L, Dimar JR, Johnson JR : Perioperative complications of lumbar instrumentation and fusion in patients with diabetes mellitus. Spine J 3 : 496-501, 2003

13. Glassman SD, Carreon L, Djurasovic M, Campbell MJ, Puno RM, Johnson JR, et al. : Posterolateral lumbar spine fusion with INFUSE bone graft. Spine J 7 : 44-49, 2007

14. Inoue K, Ohgushi H, Yoshikawa T, Okumura M, Sempuku T, Tamai S, et al. : The effect of aging on bone formation in porous hydroxyapatite : biochemical and histological analysis. J Bone Miner Res 12 : 989-994,
1997

15. Ito Z, Matsuyama Y, Sakai Y, Imagama S, Wakao N, Ando K, et al. : Bone union rate with autologous iliac bone versus local bone graft in posterior lumbar interbody fusion. Spine (Phila Pa 1976) 35 : E1101E1105, 2010

16. Kang J, An H, Hilibrand A, Yoon ST, Kavanagh E, Boden S : Grafton and local bone have comparable outcomes to iliac crest bone in instrumented single-level lumbar fusions. Spine (Phila Pa 1976) 37 : 10831091,2012

17. Ko KI, Coimbra LS, Tian C, Alblowi J, Kayal RA, Einhorn TA, et al. : Diabetes reduces mesenchymal stem cells in fracture healing through a TNFa-mediated mechanism. Diabetologia 58 : 633-642, 2015

18. Koerner JD, Yalamanchili P, Munoz W, Uko L, Chaudhary SB, Lin SS, et al. : The effects of local insulin application to lumbar spinal fusions in a rat model. Spine J 13 : 22-31, 2013

19. Lee HS, Lee JH, Lee JH : A comparison of dynamic views using plain radiographs and thin-section three-dimensional computed tomography in the evaluation of fusion after posterior lumbar interbody fusion surgery. Spine J 13 : 1200-1207, 2013

20. Lee JH, Lee KM, Baek HR, Jang SJ, Lee JH, Ryu HS : Combined effects of porous hydroxyapatite and demineralized bone matrix on bone induction : in vitro and in vivo study using a nude rat model. Biomed Mater 6 : 015008, 2011

21. Lee KJ, Roper JG, Wang JC : Demineralized bone matrix and spinal arthrodesis. Spine J 5 (6 Suppl) : 217S-223S, 2005

22. Okuyama K, Abe E, Suzuki T, Tamura Y, Chiba M, Sato K: Influence of bone mineral density on pedicle screw fixation : a study of pedicle screw fixation augmenting posterior lumbar interbody fusion in elderly patients. Spine J 1 : 402-407, 2001

23. Suetsuna F, Yokoyama T, Kenuka E, Harata S : Anterior cervical fusion using porous hydroxyapatite ceramics for cervical disc herniation. A two-year follow-up. Spine J $1: 348-357,2001$

24. Tan GH, Goss BG, Thorpe PJ, Williams RP : CT-based classification of long spinal allograft fusion. Eur Spine J 16 : 1875-1881, 2007

25. Thalgott JS, Giuffre JM, Fritts K, Timlin M, Klezl Z : Instrumented posterolateral lumbar fusion using coralline hydroxyapatite with or without demineralized bone matrix, as an adjunct to autologous bone. Spine J 1 : 131-137, 2001

26. Yi J, Lee GW, Nam WD, Han KY, Kim MH, Kang JW, et al. : A prospective randomized clinical trial comparing bone union rate following anterior cervical discectomy and fusion using a polyetheretherketone cage : hydroxyapatite/B-tricalcium phosphate mixture versus hydroxyapatite/ demineralized bone matrix mixture. Asian Spine J 9 : 30-38, 2015 